CARPATHIAN J. MATH.

Volume 38 (2022), No. 1,

Pages 13 - 19
Online version at https : //www. carpathian. cunbm. utcluj. ro/

Print Edition: ISSN 1584 - 2851; Online Edition: ISSN 1843 - 4401

DOI: https://doi.org/10.37193/CJM.2022.01.02

Dedicated to the memory of Academician Mitrofan M. Choban (1942-2021)

\title{
Unpredictability in Markov chains
}

\author{
MARAT AKHMET
}

ABSTRACT. We have formalized realizations of Markov chains as conveniently constructed sequences, and explained, why the random dynamics admits the unpredictability, the concept introduced in our papers previously. The method of the domain structured dynamics (dynamics on labels) has been applied. An illustrating example with a proper numerical simulation is provided.

\section{INTRODUCTION AND PRELIMINARIES}

The first goal of the Markov research [19] was to show that the random processes of dependent events may behave as with independent events. Thus, respectively simple models were invented, most effective for many applications. It is impossible underestimate the role of the Markov chains in development of random dynamics theory and its applications. For instance, the egrodic theorem was strictly approved at the first time under the circumstances. There are many observations that the chains are relative to symbolic dynamics and correspondingly to Bernoulli scheme. The significant step for the comprehension was done by Donald Ornstein in [21], who verified that $B$-automorphisms such as subshifts of finite type and Markov shifts, Anosov flows and Sinai's billiards, ergodic automorphisms of the $n$-torus $[22,23]$ and the continued fraction transforms are, in fact, isomorphic. Issuing from these sources it is of great interest not only to show that various random processes can be described in terms of chaos, but they relate equally in the sense to each other. Researchers have looked for chaos in random dynamics, as well as for stochastic features in deterministic motions $[12,13,14]$. Thus, the problem of chaotic ingredients in Markov chains, which is discussing in the paper, is a part of the more general project.

The unpredictable orbit [1] as a single isolated motion, presenting the Poincaré chaos [2], and identified in the chains as a certain event, is the ultimate point of comprehension of results of this paper. In other words, the main result of this paper is existence of a realization, which is an unpredictable sequence, and its closure, in the topology of convergence on bounded intervals, contains all other realizations of the random process. This implies that each finite simulation of the dynamics is an ark of the unpredictable realization. In other words, it has been proved that the unpredictability is a certain event for the Markov chain. Let us remind that an event is certain, if it occurs at every performance of an experiment.

The main task of the present research is to find signs of the unpredictability in Markov chains. Then, to show that the presence is maximal in the sense of probability. The method of domain structured chaos is originated in $[4,5]$ is in the basis of our study. In the book

Received: 26.03.2021. In revised form: 28.10.2021. Accepted: 04.11.2021

2010 Mathematics Subject Classification. 37B10, 39A33, 39A50, 60J10. chaos.

Key words and phrases. Markov chains, the unpredictability, infinite realizations, unpredictable sequences, Poincaré 
[11], we have suggested, issuing from the wide spectra of problems, call it Domain Structured Dynamics. In the present research, we suggest another term Dynamics on Labels. It has been successfully applied for chaos indication in fractals [4], neural networks [5], and for Bernoulli processes [7].

One can apply finite $[12,13,15]$ partitioning and symbolic dynamics to find randomness in deterministic motions. Differently, we utilize infinite and even uncountable partitioning $[3,4,9]$ to establish strong relations of the random processes with Poincaré chaos. We formalize a chaos generation through specially structured sets, subduing them to the abstract similarity map. This provides to us the comprehension that chaotic behavior is proper to the Bernoulli scheme dynamics and then to various random and deterministic processes on discrete and continuous time $[6,7,8]$. Presently, we are on the next step of the exploration, and show how chaos can be recognized in Markov chains. Timehomogeneous chains with a finite state space and memory are under discussion.

The approach is applied such that the dynamics can be investigated, focusing not on ergodicity, but on a single motion description such that an individual geometry is better understood. Thus, we show that an isolated realization of a Markov chain behaves in time identically with a properly chosen realization of a Bernoulli scheme, and they both are identical in dynamics with the path of the similarity map in a correspondingly chosen space.

The closure of the unpredictable realization as it is Poisson stable is said to be the quasi-minimal set [24]. It contains uncountable set of unpredictable realizations. We have proved that a quasi-minimal set as the union of all infinite realizations of the Markov chain is a certain event. In other words, each experiment of the process produces an orbit of the quasi-minimal set. This is another formulation of the main result.

Our research confirms that the Bernoulli scheme [7], Markov chains, as well as abstract hyperbolic dynamics [6] are all with the same type of chaos. It is significant that the Poincare chaos is proper for the motions. This provides, new opportunities, exceptionally for stochastic processes. We suppose that the research can be complemented with similar analysis for other Bernoulli automorphisms considered by D. Ornstein [21] as well with extension of the results for majority of stochastic processes, if proper structured domains will be constructed.

\section{MARKOV CHAINS}

A Markov chain is a stochastic model, which describes a sequence of possible events such that the probability of each event depends only on the state attained in the previous one $[17,18]$. There are many applications of the Markov chains as statistical models of real-world processes such as studying queues or lines of customers arriving at an airport, currency exchange rates, cruise control systems in motor vehicles and animal population dynamics [20].

Let a discrete-time stochastic process $X_{n}, n \geq 0$, on a countable set $S$ be given. That is, a collection of random variables defined on a probability space $(\Omega, F, P)$, where $P$ is a probability measure on a family of events $F$ in an event-space $\Omega$. The set $S$ is the state space of the process, and the value $X_{n} \in S$ is the state of the process at time $n$. The Markov chain, is a stochastic process such that the Markov property $P\left\{X_{n+1}=s_{j} \mid X_{0}, \ldots, X_{n}\right\}=$ $P\left\{X_{n+1}=s_{j} \mid X_{n}\right\}$ is true for all $s_{i}, s_{j} \in S$ and $n \geq 0$, and $P\left\{X_{n+1}=s_{j} \mid X_{n}=s_{i}\right\}=p_{i j}$, where $p_{i j}$ is the transition probability that the chain jumps from state $i$ to state $j$. The property says that, at any time $n$, the next state $X_{n+1}$ is conditionally independent of the past $X_{0}, \ldots, X_{n-1}$ given the present state $X_{n}$. More precisely, that the transition probabilities do 
not depend on the time parameter $n$. That is, the chain is time-homogeneous. If the transition probabilities were functions of time, the process would be a non-time-homogeneous Markov chain.

Consider a finite state space $S=\left\{s_{1}, \ldots, s_{m}\right\}$, where $m$ is a natural number, not smaller than two, and a metric $d$ for the space. Denote $p_{i j}=p_{j}\left(s_{i}\right)$ the Markov probability for $s_{j}$ such that $\sum_{j=1}^{m} p_{i j}=1$ for all $i=1, \ldots, m$. In what follows, we shall investigate the problem of chaos considering that all the probabilities $p_{i j}, i, j=1, \ldots, m$, are positive.

If $f_{i j}$ is the event, which consists of two elementary events, $s_{i}$ and $s_{j}$, happen successively, then an infinite realization of the Markov chain $X_{n}, n \geq 0$, can be formalized as the infinite sequence $f_{i_{1} j_{1}} f_{i_{2} j_{2}} \ldots f_{i_{n} j_{n}} \ldots$, with $i_{k}=j_{k-1}$ for all $k=2,3, \ldots$ We have that $p\left(f_{i j}\right)=p_{i j}$. The formalization does not give advantages, if one consider the chains without memory, but it makes easier the discussion of the processes with memory, in what follows.

Present the last sequence as the label $\mathcal{F}_{i_{1} i_{2} \ldots}, i_{k}=1,2, \ldots, m, k=1,2, \ldots$, of the space of labels, $\mathcal{F}$, with the metric $\delta\left(\mathcal{F}_{i_{1} i_{2} \ldots}, \mathcal{F}_{l_{1} l_{2} \ldots}\right)=\Sigma_{k=1}^{\infty} d\left(s_{i_{k}}, s_{l_{k}}\right) / 2^{k}$.

Next, we will consider Markov chains with memory of a non-zero length, besides the zero length memory. We start with the length equal to two such that the element $f_{i j k}$ presents three elementary events $s_{i}, s_{j}$ and $s_{k}$ happen successively, and the probability for $s_{k}$ is equal to $p_{i j k}=p_{k}\left(s_{i}, s_{j}\right)$. Then the Markov chain with memory has the formal presentation $f_{i_{1} j_{1} k_{1}} f_{i_{2} j_{2} k_{3}} \ldots f_{i_{n} j_{n} k_{n}} \ldots$, where $j_{l}=k_{l-1}, i_{l}=j_{l-1}$ for all $l=2,3, \ldots$ Accepting the last sequence as the element $\mathcal{F}_{i_{1} i_{2} \ldots}, i_{k}=1,2, \ldots, m, k=1,2, \ldots$, of the space $\mathcal{F}$ with the metric $\delta\left(\mathcal{F}_{i_{1} i_{2} \ldots}, \mathcal{F}_{l_{1} l_{2} \ldots}\right)=\Sigma_{k=1}^{\infty} d\left(s_{i_{k}}, s_{l_{k}}\right) / 2^{k}$ we attain the basis, common with that for the chain without memory.

At last, consider the Markov process with the memory of the length equal to arbitrary natural number $n$. We formalize the discussion with the elements $f_{i^{1}, \ldots, i^{n}}$, which consist of successive elementary events $s_{i^{1}}, \ldots, s_{i^{n}}$, such that the sequence $f_{i_{1}^{1}, \ldots, i_{1}^{n}} \ldots, f_{i_{1}^{1}, \ldots, i_{l}^{n}} \ldots$ with $i_{l}^{j}=i_{l-1}^{j+1}, j=1, \ldots, n-1, l=2,3, \ldots$, is the formalization of the chain. We obtain the structure for the dynamics research, if accept the last sequence as an element $\mathcal{F}_{i_{1}^{1} i_{2}^{1} \ldots}, i_{k}^{1}=$ $1,2, \ldots, m, \quad k=1,2, \ldots$, of the space $\mathcal{F}$, making stress on the events with the indexes $i_{k}^{1}, k=1,2, \ldots$.

It is clear that for all cases, regardless are they with memory or not, we have constructed one and the same space $\mathcal{F}$ of elements $\mathcal{F}_{i_{1} i_{2} \ldots}, i_{k}=1,2, \ldots, m, k=1,2, \ldots$, with the distance $\delta\left(\mathcal{F}_{i_{1} i_{2} \ldots}, \mathcal{F}_{l_{1} l_{2} \ldots}\right)=\Sigma_{k=1}^{\infty} d\left(s_{i_{k}}, s_{l_{k}}\right) / 2^{k}$. This is why, the space $(\mathcal{F}, \delta)$ is the main object of analysis for chaos presence in the random process, in what follows.

Complete the dynamics with the special mapping $\varphi: \mathcal{F} \rightarrow \mathcal{F}$ such that

$$
\varphi\left(\mathcal{F}_{i_{1} i_{2} \ldots i_{n} \ldots}\right)=\mathcal{F}_{i_{2} i_{3} \ldots i_{n} \ldots},
$$

for each element of the set. The map $\varphi$ is in the paradigm of the Bernoulli shift [23], known for the symbolic dynamics. It is said to be the abstract similarity map [4] as it is convenient to describe fractals, which are determined through the abstract self-similarity. The triple $(\mathcal{F}, \varphi, \delta)$ is said to be the abstract similarity dynamics [11].

From the definitions it implies that appearance of values of the map is the same as for the members of the state space, which ordered successively. Consequently, if one proves that the map is chaotic one of types in literature, then we have to recognize that the chaos is proper for the stochastic dynamics if appropriate probability arguments are provided.

The following sets,

$$
\mathcal{F}_{i_{1} i_{2} \ldots i_{n}}=\bigcup_{j_{k}=1,2, \ldots, m} \mathcal{F}_{i_{1} i_{2} \ldots i_{n} j_{1} j_{2} \ldots}
$$

where indices $i_{1}, i_{2}, \ldots, i_{n}$, are fixed, were introduced in $[4,5]$. 
It is clear that

$$
\mathcal{F} \supseteq \mathcal{F}_{i_{1}} \supseteq \mathcal{F}_{i_{1} i_{2}} \supseteq \ldots \supseteq \mathcal{F}_{i_{1} i_{2} \ldots i_{n}} \supseteq \mathcal{F}_{i_{1} i_{2} \ldots i_{n} i_{n+1}} \ldots, i_{k}=1,2, \ldots, m, k=1,2, \ldots,
$$

that is, the sets form a nested sequence.

Since one can verify that

$$
\varphi^{n}\left(\mathcal{F}_{i_{1} i_{2} \ldots i_{n}}\right)=\mathcal{F}
$$

for arbitrary natural number $n$ and $i_{k}=1,2, \ldots, m, k=1,2, \ldots$, there is a reason to call $\varphi$ a similarity map.

We will say that for the sets $\mathcal{F}_{i_{1} i_{2} \ldots i_{n}}$ the diameter condition is valid, if

$$
\sup _{i_{k}=1,2, \ldots, m} \operatorname{diam} \mathcal{F}_{i_{1} i_{2} \ldots i_{n}} \rightarrow 0 \text { as } n \rightarrow \infty,
$$

where $\operatorname{diam}(A)=\sup \{\delta(\mathbf{x}, \mathbf{y}): \mathbf{x}, \mathbf{y} \in A\}$, for a set $A$ in $\mathcal{F}$.

Define the function $\delta(A, B)=\inf \{\delta(\mathbf{x}, \mathbf{y}): \mathbf{x} \in A, \mathbf{y} \in B\}$, for two nonempty sets $A$ and $B$ in $\mathcal{F}$. Set $\mathcal{F}$ satisfies the separation condition of degree $n$ if there exist a positive number $\varepsilon_{0}$ and a natural number $n$ such that for arbitrary indices $i_{1} i_{2} \ldots i_{n}$ one can find indices $j_{1} j_{2} \ldots j_{n}$ such that

$$
\delta\left(\mathcal{F}_{i_{1} i_{2} \ldots i_{n}}, \mathcal{F}_{j_{1} j_{2} \ldots j_{n}}\right) \geq \varepsilon_{0} .
$$

Let us give the definition of the unpredictability in the sense of the dynamics $(\mathcal{F}, \delta)$.

Definition 2.1. [11] A point $\mathcal{F}_{i_{1} i_{2} \ldots}$ from the set $\mathcal{F}$ is unpredictable, if there exist a positive number $\varepsilon_{0}$ and sequences $t_{m}, s_{m}$, of natural numbers both of which diverge to infinity such that $\varphi^{t_{m}}\left(\mathcal{F}_{i_{1} i_{2} \ldots i_{k} \ldots}\right) \rightarrow \mathcal{F}_{i_{1} i_{2} \ldots i_{k} \ldots}$ as $m \rightarrow \infty$ and $\delta\left(\varphi^{t_{m}+s_{m}}\left(\mathcal{F}_{i_{1} i_{2} \ldots i_{k} \ldots}\right), \varphi^{s_{m}}\left(\mathcal{F}_{i_{1} i_{2} \ldots i_{k} \ldots}\right)\right) \geq$ $\epsilon_{0}$ for each natural number $m$.

Theorem 2.1. If the diameter and separation conditions are valid, then dynamics of the similarity map possesses an unpredictable trajectory.

Proof. Let us fix an unpredictable point of the dynamics as a member $\mathcal{F}_{i_{1} i_{2} \ldots i_{k} \ldots}$ of $\mathcal{F}$, which index sequence $i_{1} i_{2} \ldots i_{k} \ldots$ consists of subsequently chosen all strings of length one, then all possible strings of length two, etc., to infinity. The sample of this construction is seen for the two symbolic alphabet case in the next diagram,

$$
(\underbrace{01}_{\text {blocks }}|\underbrace{00011011}_{2 \text { blocks }}| \underbrace{000001010011 \ldots}_{3 \text { blocks }} \mid \ldots) .
$$

It is easily seen, by the diameter condition that the point is Poisson stable. That is, there exists a sequence of integers, $t_{m}$, divergent to infinity such that $\varphi^{t_{m}}\left(\mathcal{F}_{i_{1} i_{2} \ldots i_{k} \ldots}\right) \rightarrow$ $\mathcal{F}_{i_{1} i_{2} \ldots i_{k} \ldots}$ as $n \rightarrow \infty$. Consider the unpredictability property. We will show that there is a sequence $s_{m}$ divergent to infinity such that $\delta\left(\varphi^{t_{m}+s_{m}}\left(\mathcal{F}_{i_{1} i_{2} \ldots i_{k} \ldots}\right), \varphi^{s_{m}}\left(\mathcal{F}_{i_{1} i_{2} \ldots i_{k} \ldots}\right)\right) \geq \epsilon_{0}$, where $\epsilon_{0}$ is the separation constant for the space $(\mathcal{F}, \delta)$. Assume on the contrary, that there is no such sequence. Then, $\delta\left(\varphi^{t_{m}+i}\left(\mathcal{F}_{i_{1} i_{2} \ldots i_{k} \ldots}\right), \varphi^{i}\left(\mathcal{F}_{i_{1} i_{2} \ldots i_{k} \ldots}\right)\right)<\epsilon_{0}$, for all natural $i$. This contradicts to the diameter condition, and the choice of the initial point, since means absence of the initial string of length $n$ in the index sequence of the point $\varphi^{t_{m}}\left(\mathcal{F}_{i_{1} i_{2} \ldots i_{k} \ldots}\right)$.

It is easily seen that for a finite metric space $(S, d)$, the Definition 2.1 has the next form, which is most convenient for the present research. 
Definition 2.2. A bounded sequence $s_{i} \in S, i=0,1, \ldots$, is called unpredictable if there exist a positive number $\varepsilon_{0}$ and sequences $t_{n}, u_{n}, n=1,2, \ldots$, of positive integers both of which diverge to infinity such that $s_{i+t_{n}}=s_{i}$ for each bounded interval of integers, if $n$ is sufficiently large, and $d\left(s_{t_{n}+u_{n}}, s_{u_{n}}\right) \geq \varepsilon_{0}$ for each natural number $n$.

Let us fix an unpredictable realization of the chain, which can be determined as follows. Consider an unpredictable point $\mathcal{F}_{i_{1} i_{2} \ldots}^{*}$ of the map $\varphi$. Fix the sequence, $s^{*}=\left\{s_{i_{k}}^{*}\right\}_{k}$, which is the corresponding unpredictable realization of the Markov chain. Duo to the Definition 2.2 and Theorem 2.1, the following assertion is valid.

Theorem 2.2. Each finite realization of the Markov chain coincides with an arc of $s^{*}$. That is, the unpredictable realization happens in each experiment of the chain, and is a certain event.

Thus, one has to recognize that each experiment of the Markov chain, with and without memory, produces an arc of an unpredictable orbit. This result can be considered as the main achievement of this research. It agrees with the principle result of the ergodic theory [25] that a single trajectory proves behavior of the whole dynamics and all other trajectories. In fact, we can say that it is a fixed unpredictable orbit. In other words, this is reproduction of the chaos as a certain event. This is what guaranties the irregularity of each finite sample path of the chain.

Now, let us consider the random dynamics for deterministic sensitivity. For the discussion, let us fix an infinite sequence of elements of the state space. The probability that a simulated realization will coincide with the sequence forever is equal to zero. Consequently, the complimentary event that an experiment with the same start state will diverge from the sequence at some finite time is equal to one. Since of the metric it means that the sensitivity is present for the random dynamics with probability one.

To finalize the relationship of the stochastic dynamics and deterministic chaos, we conclude that there are elements of the deterministic chaos, which are certain events such as appearance of an unpredictable sequence as a finite realization, and there are ingredients fo chaos such as the sensitivity, which are most probable, but not deterministic, not certain events. Possibly, it is useful to say that chaos is proper for the random processes with increasing to one probability as time is increasing. In this sense, one can recommend the second law for unpredictable strings $[10,11]$.

\section{AN EXAMPLE: RANDOM WALK}

Consider the following Markov chain as an example. Let the real valued scalar dynamics $X_{n+1}=X_{n}+Y_{n}, n \geq 0$, be given such that $Y_{n}=\{-1,1\}$ is a random variable. Since we expect for the chaotic dynamics realizations to be bounded, the special chain with boundaries is constructed. That is, the probability distribution $P(1)=P(-1)=1 / 2$, if $X_{n} \neq 1,4$, and certain events $Y_{n}=-1$, if $X_{n}=4$, and $Y_{n}=1$, if $X_{n}=1$. To satisfy the construction of the present research, we will make the following agreements. First of all, denote $s_{0}=1, s_{1}=2, s_{2}=3, s_{3}=4$. Consider, the state space $S=\left\{s_{1}, s_{2}\right\}$. Introduce the following events, $f_{11}=\left\{s_{1}, s_{0}, s_{1}\right\}, f_{12}=\left\{s_{1}, s_{2}\right\}, f_{21}=\left\{s_{2}, s_{1}\right\}, f_{22}=\left\{s_{2}, s_{3}, s_{2}\right\}$. It is clear that $p_{i 1}+p_{i 2}=1, i=1,2$, and all the probabilities are equal to the half. That is, despite the differences with the theoretical chain construction in the main body of the paper, we have the sequences $f_{i_{1} j_{1}} f_{i_{2} j_{2}} \ldots f_{i_{n} j_{n}} \ldots$, with $i_{k}=j_{k-1}$ for all $k=2,3, \ldots$, and we are in the circumstances of the domain structured chaos. Consequently, there is unpredictability. To visualize an unpredictable realization, we will draw the graph of the function $\psi(t)=X_{n}$, if $t \in[n / 10,(n+1) / 10), 0 \leq t \leq 60$. According to Theorem 2.2, it is an arc of an unpredictable sequence. The graph of the function is seen in Figure 1. It illustrates an unpredictable sequence, the sample path of the random walk. 


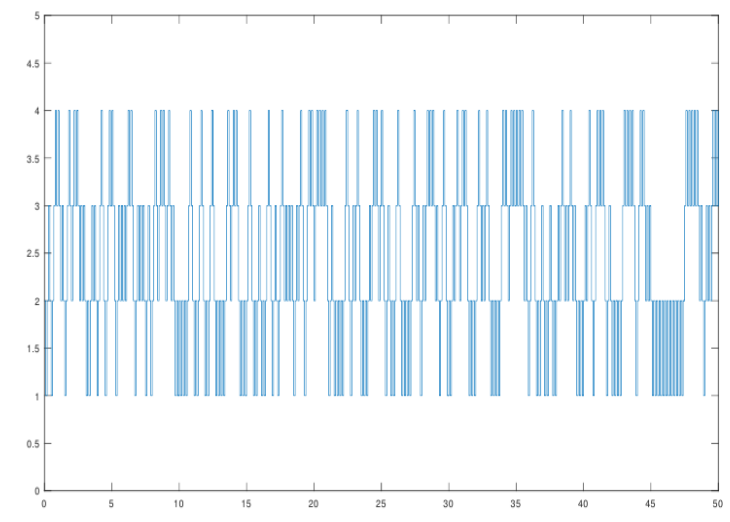

FIGURE 1. The graph of the function $\psi(t)$ as visualization of the unpredictable realization of the chain $X_{n}, n \geq 0$. The vertical lines are drawn for better visibility.

\section{CONCLUSIONS}

The outcome of the research is the existence of an unpredictable sequence as a realization of the Markov chain, and the sequence appears as finite realization of each experiment of the process. That is, appearance of the sequence is a certain event for the stochastic dynamics. In the same time the sensitivity appears not deterministically, but with probability one as time increases to infinity. From this point of view one can say that the deterministic chaos is an event for the stochastic dynamics with probability one. This result is true for many other discrete time random processes. For instance, the Bernoulli scheme. The significant use of the investigation is that one can unite methods of deterministic chaos with those for stochastic dynamics. Many other opportunities may appear. Among the methods are control and synchronization of chaos [16]. We have discussed the sensitivity is present with probability one. Evidently, it is true for the Bernoulli scheme $[10,11]$ and other dynamics, which can be approved for the similarity dynamics. Next, our study will relate Markov processes with continuous time, as well as unbounded [8, 11]. This also is connected to many other random processes. Our results provide more lights on the Markov chains as ergodic processes, since we have shown that there is the uncountable set of realizations, which are unpredictable orbits of the dynamics, and each of them is dense in the set of all realizations [2].

Acknowledgements. The author has been supported by 2247-A National Leading Researchers Program of TUBITAK, Theory of unpredictable oscillations and applications to neural networks dynamics, Turkey, N 120C138.

\section{REFERENCES}

[1] Akhmet, M.; Fen, M. O. Unpredictable points and chaos. Commun. Nonlinear Sci. Numer. Simulat. 40 (2016), 1-5.

[2] Akhmet, M.; Fen, M. O. Poincaré chaos and unpredictable functions. Commun. Nonlinear Sci. Numer. Simulat. 48 (2016), 85-94.

[3] Akhmet, M.; Fen, M. O.; Alejaily, E. M. Dynamics with chaos and fractals, Springer, 2020.

[4] Akhmet, M.; Alejaily, E. M. Abstract Similarity, Fractals and Chaos. Discrete and Continuous Dynamical Systems. Ser. B. 26 (2021), 2479-2497.

[5] Akhmet, M.; Alejaily, E. M. Domain-Structured Chaos in a Hopfield Neural Network. Int. J. Bifurc. Chaos 29 (2019), no. 14. 1950205. 
[6] Akhmet, M. Abstract Hyperbolic Chaos. Discontinuity, Nonlinearity and Complexity (in print).

[7] Akhmet, M. Domain structured chaos for discrete random processes. arXiv preprint arXiv:1912.10478, 2019

[8] Akhmet, M. Modular chaos for random processes. Discontinuity, Nonlinearity and Complexity (in print).

[9] Akhmet, M.; Alejaily, E. M. Abstract Fractals. Discontinuity, Nonlinearity and Complexity 10 (2021), no. 1, 135-142.

[10] Akhmet, M.; Tola, A. Unpredictable Strings. Kazakh Mathematical Journal 20 (2020), no. 3, 16-22.

[11] Akhmet, M., Domain structured dynamics: unpredictability, chaos, randomness, fractals, differential equations and neural networks, IOP publishing, 2021.

[12] Alekseev, V. M.; Yakobson, M. V. Symbolic dynamics and hyperbolic dynamic systems. Physics reports 75 (1981), no. 5, 287-325.

[13] Bowen, R. Markov partitions for axiom A diffeomorphisms. Amer. J. Math. 92 (1970), 725-747.

[14] Bowen, R. Equilibrium States and the Ergodic Theory of Anosov Diffeomorphisms. Springer, 1975.

[15] Bunimovich, L.; Sinai, Ya. Markov Partitions for Dispersed Billiards. Commun. Math. Phys. 78 (1980), no. 2, 247-280.

[16] Gonzáles-Miranda, J. M. Synchronization and control of chaos. Imperial College Press, 2004.

[17] Hajek, B. Random Processes for Engineers. Cambridge University Press, 2015.

[18] Karlin, S.; Taylor, H. E. A First Course in Stochastic Processes. Academic Press, 2012.

[19] Markov, A. A. Extension of the limit theorems of probability theory to a sum of variables connected in a chain. John Wiley and Sons, 1971.

[20] Meyn, S.; Tweedie, R. L. Markov Chains and Stochastic Stability, Cambridge University Press, 2009.

[21] Ornstein, D. Bernoulli shifts with the same entropy are isomorphic. Advances in Math. 4 (1970), 337-352.

[22] Ponce, G.; Varao, R., An Introduction to the Kolmogorov-Bernoulli Equivalence. Springer, 2019.

[23] Robinson, C. Dynamical Systems: Stability, Symbolic Dynamics, and Chaos. CRC Press, 1999.

[24] Sell, G. Topological dynamics and ordinary differential equations. Van Nostrand Reinhold, 1971.

[25] Walters, P. An Introduction to Ergodic Theory. Springer, 1982.

DEPARTMENT OF MATHEMATICS

Middle EAST TECHNICAL UNIVERSITY

06531, ANKARA, TURKEY

Email address: marat@metu.edu.tr 